\title{
Mini-midvastus versus medial parapatellar approach in total knee arthroplasty: difference in patient-reported outcomes measured with the Forgotten Joint Score
}

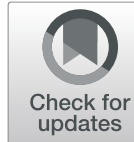

Wei Lin, Jinghui Niu, Yike Dai, Guangmin Yang, Ming Li and Fei Wang*

\begin{abstract}
Background: Low knee awareness after minimally invasive total knee arthroplasty (TKA) has become the ultimate target of a natural-feeling knee that meets patient expectations. The objective of this research was to compare the clinical outcomes of TKA via the mini-midvastus (MMV) approach or the medial parapatellar (MPP) approach, and to evaluate which approach can lead to a better quality of life after surgery.

Methods: From January 2015 to December 2016, a retrospective cohort study was conducted in 330 patients who underwent TKA via a mini-midvastus (MMV) approach. During this period, we also selected 330 patients who underwent TKA via a medial parapatellar (MPP) approach (MPP group) for comparison. Clinical results were assessed with the visual analog scale score for pain, range of motion, and the Knee Society Score. The Forgotten Joint Score was used to analyze the ability to forget the joint.

Results: There were significant differences with regard to visual analog scale score, range of motion, and the Knee Society Score until 6 months after surgery between the MMV and MPP groups $(p<0.05)$, but the differences were not significant at 12 months, 24 months, and 36 months after surgery. However, there were significant differences in the Forgotten Joint Score between the groups during the follow-up period $(p<0.05)$.

Conclusion: When forgetting the artificial joint after TKA is the ultimate target, better quality of life can be acquired by performing TKA via the MMV approach. In addition, compared with the MPP approach, the MMV approach can offer less pain and a faster recovery.
\end{abstract}

Keywords: Mini-midvastus approach, Medial parapatellar approach, Total knee arthroplasty, Forgotten Joint Score

\section{Background}

Total knee arthroplasty (TKA) is the best choice for the treatment of end-stage osteoarthritis, and it can restore knee function, relieve pain, and improve quality of life (QOL), with $95 \%$ of patients achieving a good prosthesis survival rate $[1,2]$. There are several surgical approaches

\footnotetext{
* Correspondence: doctorlinw@163.com

Department of Orthopedic Surgery, Third Hospital of Hebei Medical University, No. 139 Ziqiang Road, Shijiazhuang 050051, Hebei, People's Republic of China
}

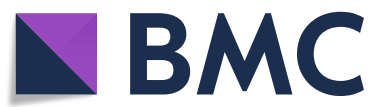

(- The Author(s). 2020 Open Access This article is licensed under a Creative Commons Attribution 4.0 International License, which permits use, sharing, adaptation, distribution and reproduction in any medium or format, as long as you give appropriate credit to the original author(s) and the source, provide a link to the Creative Commons licence, and indicate if changes were made. The images or other third party material in this article are included in the article's Creative Commons licence, unless indicated otherwise in a credit line to the material. If material is not included in the article's Creative Commons licence and your intended use is not permitted by statutory regulation or exceeds the permitted use, you will need to obtain permission directly from the copyright holder. To view a copy of this licence, visit http://creativecommons.org/licenses/by/4.0/ The Creative Commons Public Domain Dedication waiver (http://creativecommons.org/publicdomain/zero/1.0/) applies to the data made available in this article, unless otherwise stated in a credit line to the data.

for primary TKA, but it remains controversial which approach can achieve the best postoperative results.

Although the medial parapatellar (MPP) approach affords excellent surgical visualization [3], it injures the quadriceps tendon and may cause weakened extensor function, and thus, the functional outcome remains unsatisfactory [4]. To the contrary, the mini-midvastus (MMV) approach not only reduces the injury to the quadriceps, but is also associated with improved postoperative outcomes [5]. Simultaneously, the MMV 
approach has also been popularized, and compared with TKA via the standard approach, it has led to earlier postoperative flexion and a higher mean Knee Society Score (KSS) [6-8].

The traditional evaluation system often focuses on the objective evaluation of surgeons when evaluating the postoperative results of TKA. However, the concerns of patients after TKA are not always consistent with the surgeon's assessment $[9,10]$. Therefore, there is a growing tendency to use patient-reported outcome measure (PROM) tools to evaluate patient outcomes [11].

The Forgotten Joint Score (FJS) is a PROM tool designed to assess patients' ability to lose joint awareness and awareness that their joint is artificial [12]. The ability of forgetting an artificial joint has been seen as the ultimate goal of joint replacement and can reflect patient satisfaction [12, 13]. So far, few studies have evaluated the change in joint awareness after TKA via different approaches. It is important to clearly understand the actual changes in joint awareness after TKA via different approaches.

The objective of this research was to conduct a retrospective cohort study to investigate the functional outcomes of TKA via the MMV or MPP approaches using the FJS, and evaluate which approach can lead to better QOL after surgery.

\section{Methods}

After approval from the Institutional Review Committee, we performed a retrospective cohort study from January 2015 to December 2016. We included 330 patients who underwent primary TKA via the MMV approach in the MMV group. To improve the reliability of this research, we used a 1:1 ratio with regard to age, sex, body mass index (BMI), and follow-up time to select 330 patients who underwent primary TKA via the MPP approach (MPP group) for comparison.

Our eligibility criteria were (1) unilateral primary knee osteoarthritis and Kellgren and Lawrence [14] grade $\geq 3$ of the tibiofemoral joint, (2) primary cruciate-retaining TKA, (3) flexion-contracture deformity $<20^{\circ}$, and (4) varus deformity $<20^{\circ}$ [15]. Patients who had valgus or stiff knees, neurological problems, revision TKA, or previous open knee surgery were excluded.

\section{Surgical procedures}

All patients received the same anesthesia, and all surgeries were performed in our center by the same senior orthopedic surgeon. In both groups, as described by Liu et al. [16], all surgeries were performed through a midline skin incision. Using standard surgical instruments, extramedullary alignment for the tibial component and intramedullary alignment for the femoral component were obtained. In the MMV group, we dissected the vastus medialis obliquus at a distance of no more than 3 $\mathrm{cm}$ from the superior pole of the patella. In the MPP group, we performed superior extension of no more than $3 \mathrm{~cm}$ into the quadriceps tendon. The patella was subluxated laterally without eversion, and soft tissue balance was achieved in a standard method. The patellar prostheses were not replaced, and the patellar surface was only reshaped to fit the prosthesis. In all patients, we used the same cemented knee prosthesis (cruciateretaining, LINK, Hamburg, Germany, Gemini MK II). After the total knee prosthesis was implanted, the wound was closed in a standard layered fashion.

\section{Postoperative treatment}

All patients received the same postoperative pain control and rehabilitation programs [17]. After surgery, the patients were asked to walk with load-bearing as soon as possible. Functional exercises and physical therapy were best started on the first day, and active and passive extension and flexion exercises of the knee were performed for at least 3 months.

\section{Outcome measures}

Assessments were performed by a senior orthopedic surgeon who did not attend the treatments. Parameters including operative time, tourniquet time, skin incision length, and time to straight leg raise for all patients were recorded after surgery.

The visual analog scale (VAS) score for pain, range of motion (ROM), and Knee Society Score (KSS) [18] were assessed. For comparing the postoperative status of the patients who received TKA via the two different approaches, we used the Forgotten Joint Score (FJS; a 12item questionnaire with a maximum score of 100) to analyze the ability to forget the joint [12]. Higher scores represented better results. All data were assessed at 1 month, 6 months, 12 months, 24 months, and 36 months after surgery.

Standard anteroposterior and lateral radiographs were used for all preoperative and postoperative radiologic evaluations. Component and overall alignment of neutral $\pm 3^{\circ}$ was rated as satisfactory.

\section{Statistical analysis}

The normality of the continuous variables was checked with the Shapiro-Wilk test. If the data were normally distributed, the variables were checked with Student's $t$ test; if not, a non-parametric test was selected. Categorical variables were checked with Fisher's exact test or chi-square test. The correlations between the FJS and surgical approach (MMV versus MPP), sex, gender, and BMI were analyzed by multiple linear regression. The data were analyzed with SPSS 23.0 (IBM, Chicago, IL, USA). A $p<0.05$ was considered statistically significant. 


\section{Results}

All patients were followed up for at least 3 years. No significant differences were found for demographic parameters between the MMV group and the MPP group ( $p>$ 0.05) (Table 1). We found dramatic differences in skin incision length $(9.4 \pm 3.2$ versus $12.8 \pm 2.6 ; p<0.05)$ and straight leg raise $(1.3 \pm 0.7$ versus $2.8 \pm 0.6 ; p<0.05)$ between the two groups (Table 2).

The VAS, ROM, and KSS in the MMV group were better than those in the MPP group within 6 months ( $p$ $<0.05$ ), but no significant differences were found at 12 months, 24 months, and 36 months after surgery $(p>$ 0.05) (Tables 3 and 4). However, during follow-up, the FJS in the MMV group was higher than in the MPP group $(p<0.05)$ (Table 5). The multiple linear regression showed that a higher FJS was correlated with the MMV approach $(p<0.05)$ (Table 6).

According to the radiographic evaluation, there were no cases of improper implant positioning in the two groups. Until the last follow-up, no significant postoperative complications were found in the patients.

\section{Discussion}

The most important finding in our research was that when forgetting the artificial joint after TKA is the ultimate target, better QOL can be acquired by performing TKA via a MMV approach. In addition, compared with the MPP approach, the MMV approach may offer less pain and a faster recovery.

There are multiple approaches to minimally invasive TKA, including the MMV approach, the quadricepssparing approach, the mini-subvastus approach, and the limited MPP approach [19]. However, because of the difficulty of the operation, the long learning curve [7], and the difficulty of preserving the extensor mechanism [15], few surgeons currently use the quadriceps-sparing approach and the mini-subvastus approach. Thus, we used FJS to study whether the MMV approach can be successfully used routinely as a minimally invasive TKA approach.

Table 1 Patients' demographics in the two groups

\begin{tabular}{llll}
\hline Demographics & MMV group & MPP group & $p$ value \\
\hline Total patients & 330 & 330 & - \\
Age (years) & $65.2 \pm 7.7$ & $66 \pm 8.1$ & 0.08 \\
BMI $\left(\mathrm{kg} / \mathrm{m}^{2}\right)$ & $26.2 \pm 3.9$ & $25.6 \pm 3.7$ & 0.46 \\
Sex & & & 0.58 \\
$\quad$ Male & $82(24.8 \%)$ & $76(23 \%)$ & - \\
$\quad$ Female & $248(75.2 \%)$ & $254(77 \%)$ & - \\
Follow-up time (years) & $3.5 \pm 0.4$ & $3.6 \pm 0.3$ & 0.51 \\
\hline $\begin{array}{l}\text { MMV mini-midvastus, MPP medial parapatellar, BMI body mass index; mean } \pm \\
\text { standard deviation }\end{array}$
\end{tabular}

Table 2 Postoperative clinical results in the two groups

\begin{tabular}{llll}
\hline Results & MMV group & MPP group & $p$ value \\
\hline Operative time (min) & $83.1 \pm 8.4$ & $81.8 \pm 7.2$ & 0.382 \\
Tourniquet time (min) & $41.1 \pm 4.2$ & $39.2 \pm 3.4$ & 0.421 \\
Skin incision length (cm) & $9.4 \pm 3.2$ & $12.8 \pm 2.6$ & 0.032 \\
Straight leg raise (day) & $1.3 \pm 0.7$ & $2.8 \pm 0.6$ & 0.026 \\
\hline
\end{tabular}

MMV mini-midvastus, MPP medial parapatellar; mean \pm standard deviation

Several authors have attempted to compare the MMV approach and the MPP approach with conventional scores, such as the VAS, the HSS (Hospital for Special Surgery) score, and the KSS, and only found differences in short-term outcomes, but this early clinical advantage has seemed to disappear over time [5-7]. Some authors even found no differences in clinical outcomes during the follow-up period [20-22]. A more responsive jointspecific score, such as the FJS, can provide a clearer assessment of patients' postoperative satisfaction, and our study highlighted differences for the first time between the two approaches during a follow-up of at least 3 years. This shows that the FJS is an appropriate tool to evaluate patients' satisfaction, which can reflect patients' satisfaction well not only in the early postoperative period but also in the medium-term postoperative period when KSS cannot detect differences.

The FJS has been a highly rated scoring method over the last few years and is often used to measure the ability of patients to forget joint awareness or joint arthroplasty [12]. Even if the patient's knee function is improved and no pain is felt, the FJS will be lower if the patient is "aware of" the presence of their artificial joint

Table 3 The VAS and ROM in the two groups

\begin{tabular}{llll}
\hline & MMV group & MPP group & $p$ value \\
\hline VAS & & & \\
Preop & $5.1 \pm 0.7$ & $5.3 \pm 0.8$ & 0.421 \\
Postop 1 month & $3.4 \pm 0.7$ & $4.5 \pm 1.1$ & 0.032 \\
Postop 6 months & $3.1 \pm 0.8$ & $4.1 \pm 0.9$ & 0.043 \\
Postop 12 months & $2.8 \pm 1.2$ & $2.9 \pm 1.1$ & 0.072 \\
Postop 24 months & $2.3 \pm 0.8$ & $2.4 \pm 0.7$ & 0.771 \\
Postop 36 months & $2.1 \pm .7$ & $2.2 \pm 1.1$ & 0.881 \\
ROM & & & \\
Preop & $97.8 \pm 8.9$ & $97.1 \pm 6.7$ & 0.615 \\
Postop 1 month & $103.9 \pm 7.4$ & $98.6 \pm 7.3$ & 0.034 \\
Postop 6 months & $105.1 \pm 5.4$ & $100.8 \pm 7.7$ & 0.041 \\
Postop 12 months & $106.3 \pm 7.8$ & $104.1 \pm 8.2$ & 0.064 \\
Postop 24 months & $109.9 \pm 6.8$ & $108.6 \pm 7.3$ & 0.525 \\
Postop 36 months & $112.9 \pm 7.8$ & $110.2 \pm 7.2$ & 0.846 \\
\hline
\end{tabular}

$M M V$ mini-midvastus, MPP medial parapatellar, VAS visual analog score for pain; ROM range of motion; Preop preoperation, Postop postoperation; mean \pm standard deviation 
Table 4 The KSS in the two groups

\begin{tabular}{llll}
\hline & MMV group & MPP group & $p$ value \\
\hline Clinical score & & & \\
Preop & $36.5 \pm 4.8$ & $36.7 \pm 5.4$ & 0.681 \\
Postop 1 month & $71.6 \pm 5.7$ & $68.2 \pm 6.5$ & 0.032 \\
Postop 6 months & $76.3 \pm 4.9$ & $73.6 \pm 6.2$ & 0.037 \\
Postop 12 months & $81.6 \pm 5.9$ & $80.4 \pm 5.7$ & 0.087 \\
Postop 24 months & $89.6 \pm 3.2$ & $88.4 \pm 3.9$ & 0.661 \\
Postop 36 months & $93.3 \pm 4.1$ & $92.2 \pm 4.8$ & 0.783 \\
Functional score & & & \\
Preop & $38.4 \pm 3.9$ & $37.2 \pm 5.4$ & 0.783 \\
Postop 1 month & $65.1 \pm 5.9$ & $61.2 \pm 6.1$ & 0.022 \\
Postop 6 months & $71.4 \pm 4.8$ & $67.1 \pm 5.2$ & 0.033 \\
Postop 12 months & $74.1 \pm 3.1$ & $73.4 \pm 4.7$ & 0.061 \\
Postop 24 months & $82.2 \pm 3.6$ & $81.6 \pm 3.3$ & 0.511 \\
Postop 36 months & $85.1 \pm 3.7$ & $84.1 \pm 3.2$ & 0.685 \\
\hline
\end{tabular}

MMV mini-midvastus, MPP medial parapatellar, KSS Knee Society Score, Preop preoperation, Postop postoperation; mean \pm standard deviation

in daily life. As a result, minor complaints that are not identified by specific questions (such as "can you participate in sports?") are called "aware" joints, which may more sensitively reflect postoperative patient satisfaction and reduce the ceiling effect $[12,23]$. Ozaki et al. believed that the FJS is a scoring system that can express "sense of stability" as "awareness" [24]. Thomsen et al. believed that the FJS combines factors such as stiffness, pain, ability of daily activities, and patients' expectations to reflect patients' ability to forget artificial joints during activity, and therefore, this scoring system may be the best tool to evaluate the results after TKA [25]. Another study found that when using the FJS scoring system to evaluate the difference in knee awareness of patients who underwent patellofemoral arthroplasty, unicompartmental knee arthroplasty, and TKA, they found that patients who underwent different joint arthroplasties had large differences in the FJS [13].

Hiyama et al. found that quadriceps strength and pain were the main factors affecting joint awareness after TKA [26]. Quadriceps weakness is the main obstacle to patients' functional recovery after TKA, and pain is

Table 5 The FJS in the two groups

\begin{tabular}{llll}
\hline & MMV group & MPP group & $p$ value \\
\hline Postop 1 month & $57.6 \pm 6.9$ & $50.4 \pm 5.4$ & 0.027 \\
Postop 6 months & $62.4 \pm 7.1$ & $55.6 \pm 5.5$ & 0.022 \\
Postop 12 months & $71.6 \pm 5.1$ & $65.3 \pm 4.8$ & 0.041 \\
Postop 24 months & $78.6 \pm 6.3$ & $70.4 \pm 6.1$ & 0.037 \\
Postop 36 months & $81.1 \pm 4.1$ & $78.2 \pm 4.4$ & 0.046 \\
\hline
\end{tabular}

MMV mini-midvastus, MPP medial parapatellar, FJS Forgotten Joint Score, Preop preoperation, Postop postoperation; mean \pm standard deviation
Table 6 Multiple linear regression analysis

\begin{tabular}{llll}
\hline & Coefficient & $95 \%$ Cl & $p$ value \\
\hline MMV approach & 42.3 & 28.4 to 72.5 & 0.037 \\
MPP approach & 32.5 & 20.4 to 53.6 & 0.463 \\
Age & 0.903 & 0.128 to 1.431 & 0.537 \\
BMl & -0.701 & -1.814 to -0.831 & 0.974 \\
Sex & 0.857 & -1.934 to 4.547 & 0.541 \\
\hline
\end{tabular}

$M M V$ mini-midvastus, MPP medial parapatellar, $B M I$ body mass index, $\mathrm{Cl}$ confidence interval

usually one of the main criteria for success or failure after TKA. Quadriceps weakness and pain are closely related to disability [27], patient satisfaction [28], and QOL [29, 30].

One of the greatest advantages of TKA via the MMV approach is that it retains the extensor mechanism as much as possible during the operation. Therefore, it can reduce the perioperative pain and help the patients recover quickly. However, it has been pointed out that the standard MPP approach may decrease the strength of the quadriceps measured by isokinetics as much as $30.7 \%$ in the 2 years after TKA, and excessive damage to the extensor mechanism may be permanent [31]. Retaining the extensor mechanism as much as possible was the main reason why the MMV approach enabled patients to achieve faster functional recovery and higher satisfaction $[8,32]$. Our study adds to these findings by investigating the effects of quadriceps weakness and pain on joint awareness after TKA.

Some studies indicated that the performance of a postoperative straight leg raise reflects the recovery of quadriceps muscle strength [33, 34]. Schroer and Nestor measured the pre- and postoperative muscle strength of their patients who underwent TKA via the MMV approach and reported that patients had regained their preoperative quadriceps muscle strength in a short period, and even exceeded those levels by $30 \%$ at 3 to 6 months $[35,36]$. Similar results were found in our research. In the present study, the time to be able to perform a postoperative straight leg raise in patients who underwent TKA via the MMV approach was much earlier than in patients who underwent TKA via the MPP approach. This difference in quadriceps muscle strength is essential for patients to resume daily activities. As reported in previous studies $[37,38]$, we also found that the MMV approach can shorten the length of the skin incision compared with the traditional MPP approach. In addition, a shorter skin incision may produce a better esthetic effect, which can improve patients' satisfaction.

However, it has been pointed out that during the TKA with minimally invasive surgery, such as the MMV approach, the complex manipulation and poor exposure can lead to malalignment of the components [34, 39], 
which may lead to failure of TKA [40]. However, in our study, no significant differences in postoperative complications were found in the patients until the final followup. Consequently, the MMV approach that protected the extensor mechanism may be a good choice in TKA.

The limitation of this study was that it had a retrospective mid-term follow-up design. Prospective and longer-term studies should be performed to confirm these findings. In addition, further study is needed to determine the minimal clinically important difference (MCID) threshold of FKS between the two groups.

\section{Conclusion}

When forgetting the artificial joint after TKA is the ultimate target, better quality of life can be achieved by performing a TKA via the MMV approach. In addition, compared with the MPP approach, the MMV approach may offer less postoperative pain and a faster recovery.

\section{Abbreviations}

TKA: Total knee arthroplasty; MMV: Mini-midvastus; MPP: Medial parapatellar; PROM: Patient-reported outcome measure; QOL: Quality of life; ROM: Range of motion; KSS: Knee Society Score; FJS: Forgotten Joint Score; BMI: Body mass index

\section{Acknowledgements}

The authors would like to thank all the staff of the participating departments. We thank Peter Mittwede, MD, PhD, from Liwen Bianji, Edanz Editing China (www.liwenbianji.cn/ac), for editing the English text of a draft of this manuscript.

\section{Authors' contributions}

FW designed the study. WL, JHN, and YKD performed the experimental work. GMY, ML, and WL evaluated the data. WL wrote the manuscript. All authors read and approved the final manuscript.

\section{Funding}

Not applicable.

\section{Availability of data and materials}

The detailed data and materials of this study are available from the corresponding author via e-mail on reasonable request.

\section{Ethics approval and consent to participate}

This study was approved by the Third Hospital of Hebei Medical University and followed the Declaration of Helsinki. Informed consent was received from all patients

\section{Consent for publication}

Not applicable.

\section{Competing interests}

The authors declare that they have no competing interests.

Received: 10 March 2020 Accepted: 6 August 2020

Published online: 17 August 2020

\section{References}

1. Gandhi R, Dhotar H, Razak F, Mahomed NN. Predicting the longer term outcomes of total knee arthroplasty. Knee. 2010;17(1):15-8.

2. Pivec R, Issa K, Kester M, Mont MA. Long-term outcomes of MUA for stiffness in primary TKA. J Knee Surg. 2013;26(6):405-10.

3. Weinhardt C, Barisic M, Bergmann EG, Heller KD. Early results of subvastus versus medial parapatellar approach in primary total knee arthroplasty. Arch Orthop Trauma Surg. 2004;124(6):401-3.
4. Boerger TO, Aglietti P, Mondanelli N, Sensi L. Mini-subvastus versus medial parapatellar approach in total knee arthroplasty. Clin Orthop Relat Res. 2005 440:82-7.

5. Haas SB, Cook S, Beksac B. Minimally invasive total knee replacement through a mini midvastus approach: a comparative study. Clin Orthop Relat Res. 2004:428:68-73.

6. Laskin RS, Beksac B, Phongjunakorn A, Petersen M. Minimally invasive total knee replacement through a mini-midvastus incision: an outcome study. Clin Orthop Relat Res. 2004;428:74-81.

7. Haas SB, Manitta MA, Burdick P. Minimally invasive total knee arthroplasty: the mini midvastus approach. Clin Orthop Relat Res. 2006:452:112-6.

8. Karachalios T, Giotikas D, Roidis N, Malizos KN. Total knee replacement performed with either a mini-midvastus or a standard approach: a prospective randomized clinical and radiological trial. J Bone Joint Surg (Br). 2008:90(5):584-91.

9. Bullens PH, van Loon CJ, de Waal Malefijt MC, Veth RP. Patient satisfaction after total knee arthroplasty: a comparison between subjective and objective outcome assessments. J Arthroplast. 2001;16(6):740-7.

10. Janse AJ, Gemke RJ, Uiterwaal CS, Sinnema G. Quality of life: patients and doctors don't always agree: a meta-analysis. J Clin Epidemiol. 2004;57(7): 653-61.

11. Cholewinski P, Putman S, Vasseur L, Pasquier G. Long-term outcomes of primary constrained condylar knee arthroplasty. Orthop Traumatol Surg Res. 2015;101(4):449-54.

12. Behrend H, Giesinger K, Giesinger JM, Kuster MS. The "forgotten joint" as the ultimate goal in joint arthroplasty: validation of a new patient-reported outcome measure. J Arthroplast. 2012;27:430-6.e1.

13. Thienpont E, Opsomer G, Koninckx A, Houssiau F. Joint awareness in different types of knee arthroplasty evaluated with the Forgotten Joint score. J Arthroplast. 2014:29:48-51.

14. Kellgren JH, Lawrence JS. Radiological assessment of osteoarthrosis. Ann Rheum Dis. 1957:16(4):494-502.

15. Lee DH, Choi J, Nha KW, Kim HJ, Han SB. No difference in early functional outcomes for mini-midvastus and limited medial parapatellar approaches in navigation-assisted total knee arthroplasty: a prospective randomized clinical trial. Knee Surg Sports Traumatol Arthrosc. 2011; 19(1):66-73

16. Liu H, Mei X, Zhang Z, Sun J. Mini-midvastus versus mini-medial parapatellar approach in simultaneous bilateral total knee arthroplasty with 24-month follow-up. Acta Orthop Traumatol Turc. 2015:49(6):586-92.

17. Husted H, Holm G, Jacobsen S. Predictors of length of stay and patient satisfaction after hip and knee replacement surgery: fast-track experience in 712 patients. Acta Orthop. 2008;79:168-73.

18. Insall JN, Dorr LD, Scott RD, Scott WN. Rationale of the knee society clinical rating system. Clin Orthop Relat Res. 1989;248:13-4.

19. Tenholder M, Clarke HD, Scuderi GR. Minimal-incision total knee arthroplasty: the early clinical experience. Clin Orthop Relat Res. 2005:440: $67-76$.

20. Zhang Z, Zhu W, Gu B, Chen C. Mini-midvastus versus mini-medial parapatellar approach in total knee arthroplasty: a prospective, randomized study. Arch Orthop Trauma Surg. 2013:133(3):389-95.

21. Heekin RD, Fokin AA. Mini-midvastus versus mini-medial parapatellar approach for minimally invasive total knee arthroplasty: outcomes pendulum is at equilibrium. J Arthroplast. 2014;29(2):339-42.

22. Nestor BJ, Toulson CE, Backus S, Windsor RE. Mini-midvastus vs standard medial parapatellar approach: a prospective, randomized, double-blinded study in patients undergoing bilateral total knee arthroplasty. J Arthroplast. 2010;25(6 Suppl):5-11 11.e1.

23. Hamilton DF, Giesinger JM, MacDonald DJ, Giesinger K. Responsiveness and ceiling effects of the Forgotten Joint Score-12 following total hip arthroplasty. Bone Joint Res. 2016;5(3):87-91.

24. Ozaki Y, Baba T, Kaneko K, et al. Posterior versus direct anterior approach in total hip arthroplasty: difference in patient-reported outcomes measured with the Forgotten Joint Score-12. SICOT J. 2018:4:54.

25. Thomsen MG, Latifi R, Kallemose T, Husted H, Troelsen A. Does knee awareness differ between different knee arthroplasty prostheses? A matched, case-control, cross-sectional study. BMC Musculoskelet Disord. 2016:01(17):141.

26. Hiyama Y, Wada O, Nakakita S, Mizuno K. Joint awareness after total knee arthroplasty is affected by pain and quadriceps strength. Orthop Traumatol Surg Res. 2016;102(4):435-9. 
27. Mizner RL, Petterson SC, Snyder-Mackler L. Quadriceps strength and the time course of functional recovery after total knee arthroplasty. J Orthop Sports Phys Ther. 2005;35:424-36.

28. Baker PN, van der Meulen JH, Lewsey J, Gregg PJ, National Joint Registry for England and Wales. The role of pain and function in determining patient satisfaction after total knee replacement. Data from the National Joint Registry for England and Wales. J Bone Joint Surg (Br). 2007;89:893-900.

29. Tungtrongjit $Y$, Weingkum $P$, Saunkool $P$. The effect of preoperative quadriceps exercise on functional outcome after total knee arthroplasty. J Med Assoc Thail. 2012;95(Suppl. 10):S58-66.

30. Wu CL, Naqibuddin M, Rowlingson AJ, Lietman SA, Jermyn RM, Fleisher LA. The effect of pain on health-related quality of life in the immediate postoperative period. Anesth Analg. 2003;97(4):1078-85.

31. Kolisek FR, Bonutti PM, Hozack WJ, Rothman RH. Clinical experience using a minimally invasive surgical approach for total knee arthroplasty: early results of a prospective randomized study compared to a standard approach. J Arthroplast. 2007;22(1):8-13.

32. Thienpont E, Zorman D. Higher forgotten joint score for fixed-bearing than for mobile-bearing total knee arthroplasty. Knee Surg Sports Traumatol Arthrosc. 2016;24(8):2641-5.

33. Egocheaga JRV, Suarez MAS, Villan MF, Sastre VG, Gomez JRV, Merchan CR. Minimally invasive subvastus approach: improving the results of total knee arthroplasty: a prospective, randomised trial. Clin Orthop Relat Res. 2010;468: 1200-8.

34. Cheng T, Liu T, Zhang G, Peng X, Zhang X. Does minimally invasive surgery improve short term recovery in total knee arthroplasty? Clin Orthop Relat Res. 2010;468:1635-48.

35. Nestor MJ, Toulson CE, Backus SI, Lyman SL, Foote KL, Windsor RE. Minimidvastus vs standard medial parapatellar approach: a prospective, randomised, double-blinded study in patients undergoing bilateral total knee arthroplasty. J Arthroplast. 2010;25(6 Suppl):5-11.

36. Karpman RR, Smith HL. Comparison of the early results of minimally invasive vs standard approaches to total knee arthroplasty: a prospective, randomised study. J Arthroplast. 2009;24:681-8.

37. Pan WM, Li XG, Tang TS, Zhang CM. Mini-subvastus versus a standard approach in total knee arthroplasty: a prospective, randomized, controlled study. J Int Med Res. 2010:38(3):890-900

38. Thiengwittayaporn S, Kanjanapiboonwong A, Junsee D. Midterm outcomes of electromagnetic computer-assisted navigation in minimally invasive total knee arthroplasty. J Orthop Surg Res. 2013;25(8):37.

39. Dalury DF, Dennis. Mini-incision total knee arthroplasty can increase risk of component malalignment. Clin Orthop Relat Res. 2005;440:77-81.

40. Fehring TK, Odum S, Griffin WL, Nadaud M. Early failures in total knee arthroplasty. Clin Orthop Relat Res. 2001:392:315-8.

\section{Publisher's Note}

Springer Nature remains neutral with regard to jurisdictional claims in published maps and institutional affiliations.

Ready to submit your research? Choose BMC and benefit from:

- fast, convenient online submission

- thorough peer review by experienced researchers in your field

- rapid publication on acceptance

- support for research data, including large and complex data types

- gold Open Access which fosters wider collaboration and increased citations

- maximum visibility for your research: over $100 \mathrm{M}$ website views per year

At $\mathrm{BMC}$, research is always in progress.

Learn more biomedcentral.com/submissions 